peak on the seventh and eighth days while discharges reached their peak on the sixteenth and seventeenth days.

Lastly, acknowledgments are made to Dr. V. D. Allison for the bacteriological examinations and reports, and to Dr. T. H. Whittington, Consulting Oculist to the L.C.C., who visited the "Exmouth" during the epidemic and approved of what was being done.

\title{
GLAUCOMA AND LOW TENSION DISC CUP ENLARGEMENTS
}

BY

\section{RANSOM PICKARD}

EXETER

At the present time some interest is being taken in cases which present all the symptoms of glaucoma except that of increased tension. In April, 1930, I read a paper (a) dealing with this group of cases, showing that, as far as the tests employed were concerned, there was no difference from glaucoma except that of tension. Prof. F. H. Newman, D.Sc., Professor of Physics in the University College of the South-West, afterwards kindly examined the paper and the charts on which it was founded. He agreed that on the facts as presented to him the deductions were correct. He suggested that a plot of the tension against the size of the optic disc cup be made and that the elasticity of the cup should be investigated. The present paper embodies these results, an enquiry into the origin of the low tension cases, compares the clinical course of the two conditions, and discusses the name to be applied to the condition when it occurs with no rise of intra-ocular tension.

The cubic capacity was calculated upon the basis that the transverse diameter of the disc is $1.5 \mathrm{~mm}$., the vertical $1.6 \mathrm{~mm}$. and that a depth of $-3 \mathrm{D}$. equals $1 \mathrm{~mm}$.; the drawings of the discs made in my notes supplying the remaining details. It should be noted that, because of some details being absent, some cases used in the paper ( $a$ ) had to be rejected, while as many of the cases shown in it as " too advanced for use in charts" as possible were added, as the present paper does not deal with the fields of vision, and they are necessary for a more complete comparison of the clinical course.

\begin{tabular}{llcccc} 
& \multicolumn{2}{c}{$\begin{array}{c}\text { No. of eyes } \\
\text { from paper }(a)\end{array}$} & $\begin{array}{c}\text { No. of advanced } \\
\text { eyes added. }\end{array}$ & Total. \\
Glaucoma & $\ldots$ & 32 & 12 & $=$ & 44 \\
Low tension & $\ldots$ & 35 & 2 & $=$ & 37
\end{tabular}


In Charts I and II the contents above $2: 20 \mathrm{c} . \mathrm{mm}$. are shown in figures, not in true ordinate position, because of space. The tension is correctly shown in these large cups.

Tension and cubic contents of cup.-It is to be noticed in the low tension cases that 26 out of 37 have T. 20 or less; that is, the tension is generally rather low than near the border line $(27 \mathrm{~mm}$. $\mathrm{Hg}$ ), the mean for the whole group being $18 \cdot 25$; corresponding

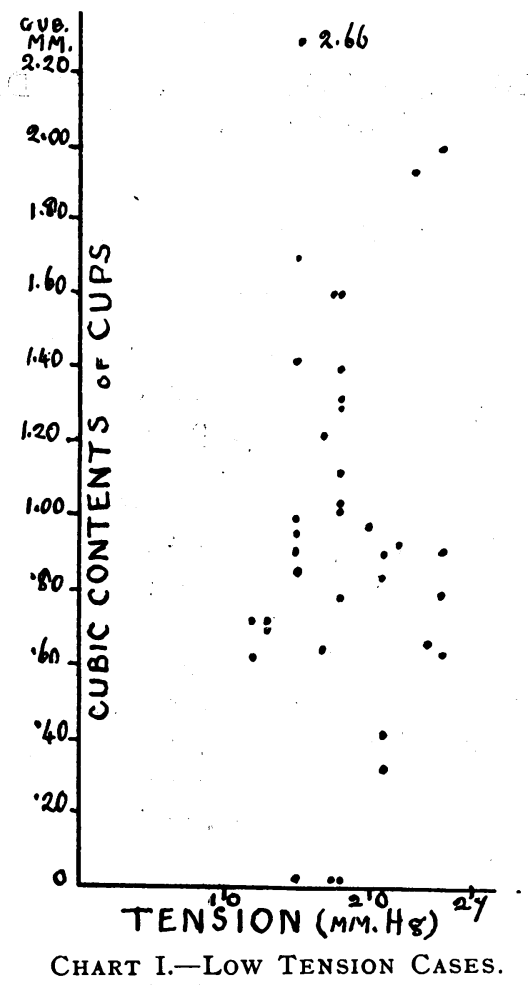

with, on the Schiötz scale, a weight of $5.5 \mathrm{gcms}$. and a deviation of $5^{\circ}$. Out of 15 cases having a cup of $1 \mathrm{c} . \mathrm{mm}$., or upwards, 13 have a tension of $18 \mathrm{~mm}$. Hg or lower; i.e., the larger cups have a lower tension.

Thus it will be seen that this chart negatives the opinion that intra-ocular pressure is any active factor in the enlargement of the cup; if this had been so, the larger cups would have had a pressure approaching the higher limits of normality; this is not the case.

As compared with the glaucoma chart, it will be seen that there are few cases with small cups. Although all cases start from a normal condition, in practice it is an enlarged cup which leads to an 
Glaucoma and Low Tension Disc Cup Enlargements 325

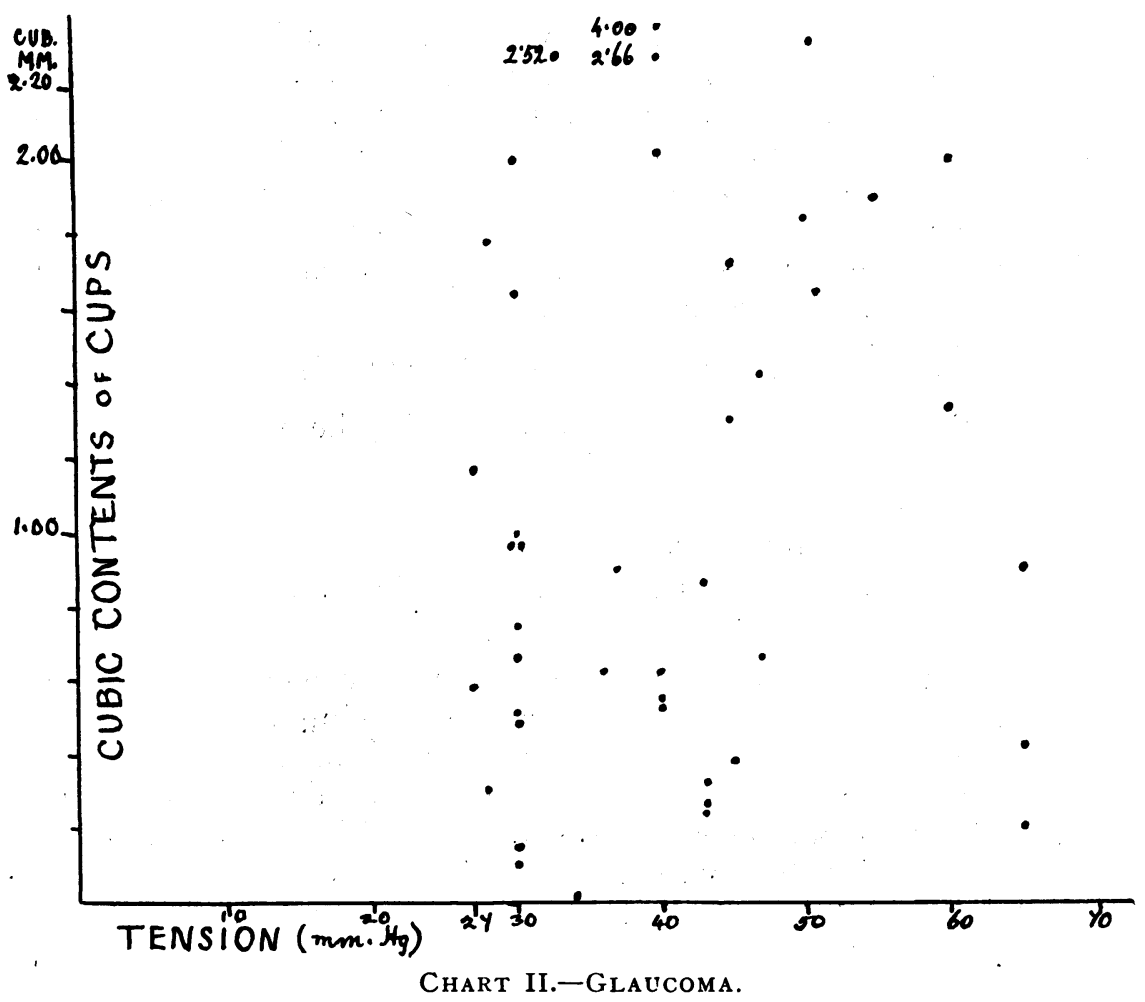

investigation ; only in the event of one large cup and the systematic examination of both fields, is an early case with small cup likely to be detected. In point of fact most patients had both disc cups enlarged when first seen. ( $(a) .17$ out of 21 patients). A third point is that the cup sizes are mostly grouped between 0.50 c.c. and 1.5 c.c.; it is exceptional for the cups to be excessively large.

Of the glaucoma cases 14 out $^{-}$of 43 have a tension between $27 \mathrm{~mm}$. and $30 \mathrm{~mm}$. $\mathrm{Hg}$, their cup sizes showing a wide distribution. The remaining 29 cases are sparsely scattered over a wide range up to $65 \mathrm{~mm}$. $\mathrm{Hg}$. Of the 15 cases with a tension of 45 or upwards, 10 have a cubic capacity of over $1 \mathrm{c} . \mathrm{mm}$.

The cases may be classified thus according to cup sizes :-

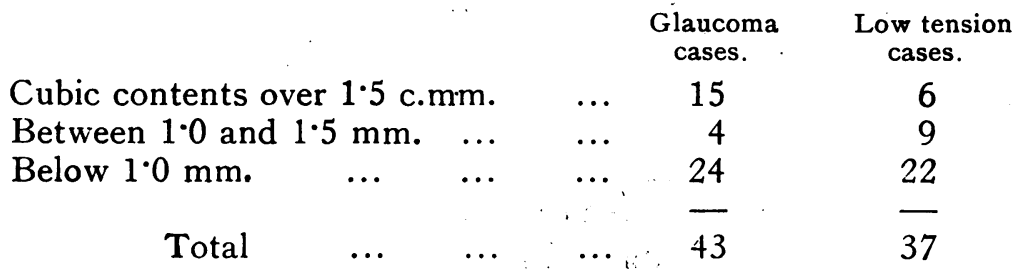


The glaucoma cases chart may be taken as illustrating the general clinical experience that much effect may be produced by a slight but constant rise in tension, though the extremely large cups are mostly caused by a high rise of tension. But the size of a cup and the tension will depend upon the stage at which the case is first seen. The group of large cups is a graphic illustration of the clinical experience that so many cases of glaucoma first seek advice with one eye in an advanced stage.

Elasticity of cup surface.-Prof. Newman suggested that the formula $S\left(\frac{1}{r^{1}}+\frac{1}{r^{2}}\right)=t$ should be used, where

$$
\begin{aligned}
& \mathrm{s}=\text { surface tension. } \\
& \mathrm{r}^{1}=\text { radius of cup in depth in } \mathrm{mm} \text {. } \\
& \mathrm{r}^{2}=\text {, ", , horizontal diameter in } \mathrm{mm} \text {. } \\
& \mathrm{t}=\text { intra-ocular tension, }
\end{aligned}
$$

the formula being used as if it were true for elasticity. Error is introduced by the bottoms of the cups not being perfect curves; but as the two groups are alike in this, it is assumed that the errors will not spoil the comparison of the groups. The figures given for elasticity are not absolute measurements of force; but these are not necessary for the comparison, as the figures in both groups would have to be multiplied by the same constants to obtain absolute measurements.

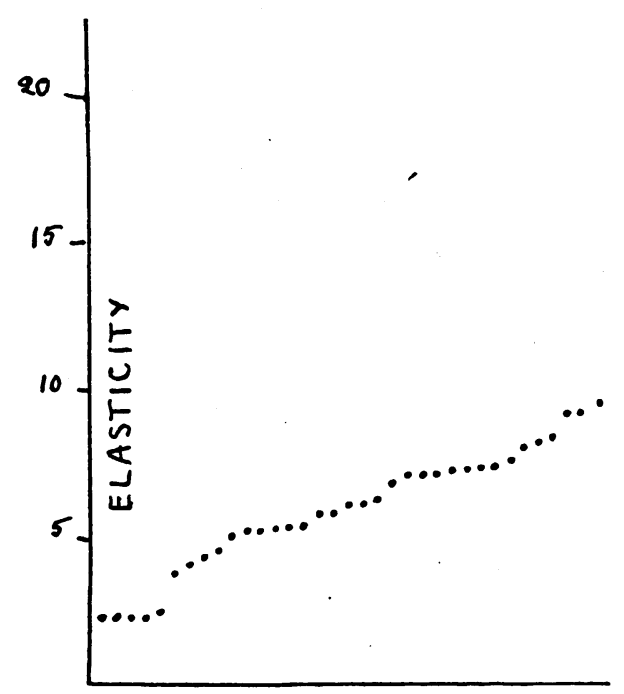

CASES

Chart III.-Low Tension. 


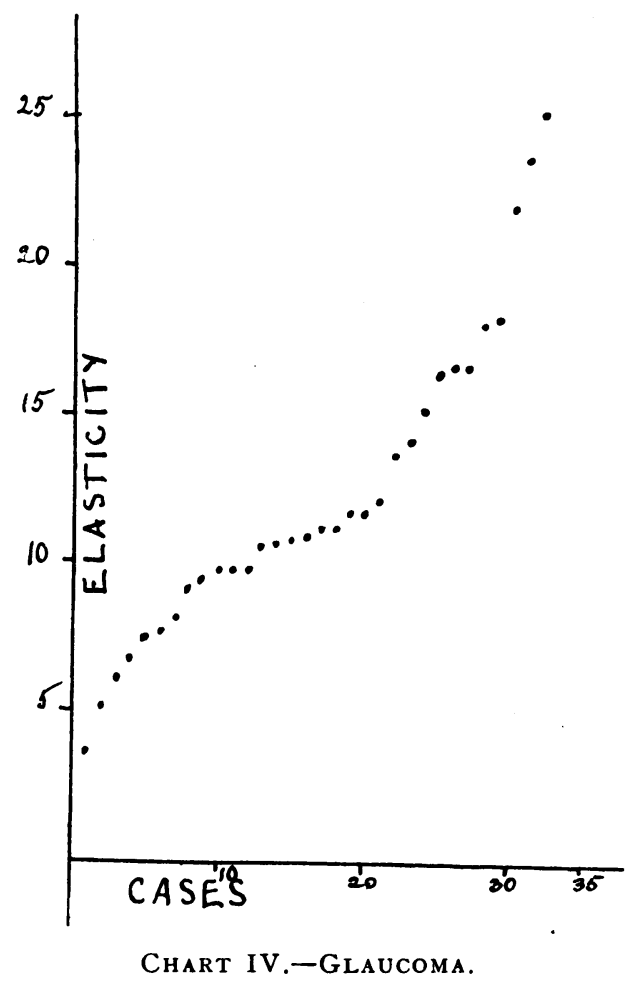

The obvious feature in the graphs thus made is the much higher elasticity in the glaucoma group. The averages for the groups are :-

Glaucoma $12 \cdot 41$

Low 'Tension $5 \cdot 84$

Using general terms, the elasticity in the glaucoma group is rather more than double that of the low tension group.

These charts point out that there is a great variation in the resistance of the nerve head in otherwise apparently normal nerves, so that more or less equal tensions may produce very different results in different eyes. But III and IV show such differences in elasticity as can only be explained by very different conditions in the tissues of the nerve heads of the two groups. All the 35 eyes of the low tension group give a figure under 10 , of the 32 glaucoma cases in the Chart IV only 12 eyes are under 10 .

It has been suggested that the group of low tension cases may be accounted for by the supposition that they normally start with a tension much below the average and to which the eyeball is adapted and that upon these conditions supervene one or other of the causes of rise of tension. It is argued that the resultant 
increased tension will have the same effect upon the optic disc in such cases as in ordinary glaucoma; i.e., that the low tension group is a glaucomatous group with the initial balance of forces set, as it were, on a lower scale, into which as a disturbing element comes an increase of tension which, though bringing the total to less than $27 \mathrm{~mm}$. $\mathrm{Hg}$ (Schiötz) is sufficient to cause glaucoma.

There are three conditions assumed in this argument, all of which have to occur together, a group of eyes with (i) tensions much lower than the average (ii) optic discs of weaker resistance than the average (iii) a rise of tension enough to produce glaucoma but not reaching to $27 \mathrm{~mm}$. $\mathrm{Hg}$.

If the glaucoma and intermittent glaucoma groups in the paper of April, 1930 (a) be taken together, the total is 36 individuals, as contrasted with 21 for the low tension group. Seeing that this represents the result of consecutive investigation upon over 4,000 private patients, the figures may be taken as representing to a moderate degree of accuracy the incidence of these two groups in the south-west district of England, ethnologically mostly AngloSaxon with an admixture of Celtic and Norman strains; the proportion being roughly two glaucomas to one low tension case.

Cridland (ref. d.) states that in a group of 1,001 normal cases the tension was under 15 in 1.7 per cent., which would give 68 of such cases in 4,000 people, or 136 eyes.

If it be for the moment assumed that the glaucoma and low tension cases are all due to an increase of tension, then the incidence of pathological increase is, $36+21(=5 \tau)$ per 4,000 cases, 1.42 per cent. As there is no evidence that low tension eyes are more liable to increases of tension than those of average tension, we are entitled to assume that they will have an average liability to an increase. Thus, the 136 eyes of tension under $15 \mathrm{~mm} . \mathrm{Hg}$ would have a liability to a pathological increase of tension in 1.42 per cent. of eyes, equal to two eyes in the group of 4,000 people; whereas 21 cases, comprising 38 affected eyes, were found (Chart I), in which four eyes were under $15 \mathrm{~mm}$. and eight had a tension of $15 \mathrm{~mm}$.

\section{SUMMARY OF ARGUMENT}

Normal tension under $15 \mathrm{~mm}$. $\mathrm{Hg}$ at 1.7 per cent. in 4,000 cases $=136$ eyes (68 cases).

Total No. of cases in paper $(a)$

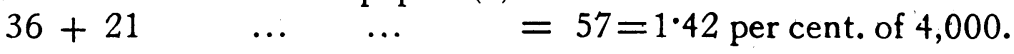

1.42 per cent. of $136 \quad \ldots=2$ eyes.

Actual No. of eyes affected yet having low tension $\ldots \quad=38$ (21 cases), 
If this calculation be accepted, it will be obvious that an increase of a previously low tension cannot account for the number of cases found, even on the widest allowances for variations in different series of cases.

It will be seen that no account has been taken of the variability in the normal resistance of the optic disc in this statistical argument. That this exists is a matter of every-day experience. Chart II may be taken as a graphic illustration of its effect in glaucoma on the size of the cups under similar tensions. It introduces another variable into the calculation, which would act both in the low tension cases as well as in glaucoma; but since the former only comprise $1 \cdot \tau$ per cent. $(d)$, even if all these had discs of low resistance the figures just given would not be affected; whereas, if less than 100 per cent. have weak optic discs, this would decrease the number which give way under an increased but low tension.

These considerations may be looked at from another point of view. Do the tensions (Chart I) of the low tension group represent an average normal group? The following is an analysis of them :-

$\begin{array}{llllcc} & & & \text { Percentage. } & \text { Cridland }(d) \text {. } \\ \text { No. under } 15 \mathrm{~mm} . \ldots & \ldots & 4 & 10.8 & 1.7 \\ \text { No. at } 15 \mathrm{~mm} . & \ldots & \ldots & 8 & 21.6 & \\ \text { Average for whole group } & \ldots & 18.25 & & 20.5\end{array}$

If the tensions as a whole were raised, the average would be raised; it is slightly below Cridland's figures, a difference such as might be expected in two different series of cases. The low tension cases are an average group, with no suggestion that they are raised within the normal limit.

This aspect may be thus summed up. The view that the cases of low tension start from a lower level involves three coincidences, a low tension, weak disc head, and an increase of tension in the same case. The alternative explanation, that there is a pathological condition of the nerve head leading to contraction, requires this one assumption and no coincidence at all.

The modus operandi.- The bearing of the graphs, in conjunction with those of my previous paper $(a)$ upon the causation of the low tension group has now to be considered.

(i) The evidence is against intra-ocular tension being the cause of the cup recession.

(ii) The lower elasticity in this group suggests an actual decrease in the resistance of the nerve head.

(iii) The group of small cup cases, found in the routine examination of the companion eyes, shows that changes have occurred in these optic discs before any cup alterations have occurred. This 
is further confirmed by a case described by me in a paper (b. Case E) on a group of low tension cases in which a companion eye, healthy to inspection, showed a much enlarged blind spot.

The right disc in this case is now 100 per cent. and $3 \mathrm{D}$. deep with an unchanged field (April, 1931.)

(iv) Any alteration in the position of a limiting surface fixed at its periphery can only occur as the result of a disturbance of the forces acting upon either side. In the case of the optic disc cup, the cup will enlarge by pressure exerted through the vitreous or by traction by the nerve head tissues; the former has been shown not to be increased, therefore the enlargement is caused by contraction of the nerve tissues.

The conclusion arrived at is that the primary cause is in the nerve head itself, and is not an effect inflicted upon it from without, as in the case in glaucoma. It apparently sometimes causes diminution of the $1 / 1000$ white field and enlargement of the blind spot before the cup is enlarged. Later, the primary cause leads to recession of the nerve head and enlargement of the disc cup, with consequent effects on the nerve bundles.

The next question is how the cup enlargement acts upon the nerve fibres, causing field contractions like those in glaucoma.

In glaucoma there are two possibilities, longitudinal lengthening of the fibres by the enlargement of the disc cup; and lateral pressure on them through the vitreous, the tissues of the nerve head acting as a counterpressure. The longitudinal lengthening caused by the enlarged cup would act equally in the low tension group, but not the lateral pressure.

\section{CUP SECTIONS}

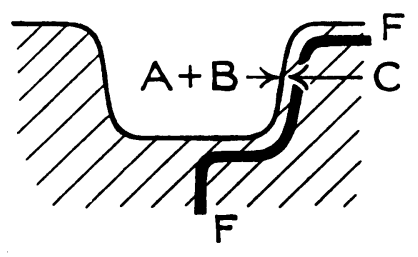

GLAUCOMA

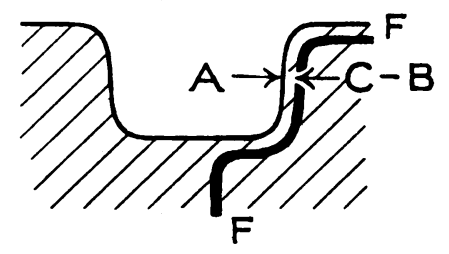

LOW TENSION

In the two diagrams $\mathrm{FF}=$ a nerve bundle in the nerve head.

$\mathrm{A}=$ Normal tension of the eye.

$\mathrm{B}=$ Increase of tension in glaucoma or retraction in the low tension group.

$\mathrm{C}=$ Counterbalancing external tension (i.e., in nerve head.)

(1) Normally B is absent and A and C are in equilibrium. 
(2) In glaucoma $\mathrm{B}$ has both a longitudinal and transverse action on the nerve head fibres; a stretching and a crushing action.

(3) In the low tension group the counterpressure $\mathrm{C}$ is lowered by $\mathrm{B}$, so that $\mathrm{A}$ cannot exerc the lateral pressure as in glaucoma, for though it exceeds $C-B$ by the amount $B$, it has a lowered resistance against which to compress the nerve bundle FF.

If this reasoning is correct, the effect of the cup on the nerve fibres, as far as the enlargement is concerned, is limited to changes caused by the longitudinal tension on the fibres. It must be noted that this does not invalidate the possibility of effects on the nerve fibres produced by the original cause of the recession, to which reference has already been made, and which effects would continue as long as contraction of the tissues of the nerve head continues.

The clinical course.-In chronic non-intermittent glaucoma it may fairly be stated that blindness is the final inevitable result if the patient lives long enough, a rule with very few exceptions. On a priori grounds it may be surmised that this might not be so in the low tension group. If the recession be considered as due to a contraction of the nerve head tissues, it will be seen that the amount of this might vary, giving rise to different degrees in the final size of the cup and of effect upon central vision and field. The following are reasons for thinking this is so.

(i) In the paper (a) while out of 21 patients in the low tension group 4 had one eye too advanced to be used in constructing the charts, out of 36 patients in the glaucoma and intermittent glaucoma groups 21 had one eye too advanced for use in the chart. In groups of these small numbers minor differences would not be more than would be expected, but a disproportion such as this is significant ; it is a fair deduction that in the group of low tension cases the affection tends to stop short of severe loss of the visual field.

\begin{tabular}{lcccc} 
& \multicolumn{3}{c}{ Glaucoma. } & Low tension. \\
Total affected eyes & $\ldots$ & 67 & 40 \\
Analysis of & severe cases : & & \\
Blind ... & $\ldots$ & $\ldots$ & 5 & 0 \\
V $1 / 60$ & $\ldots$ & $\ldots$ & 5 & 1 \\
Fields very small & $\ldots$ & 11 & 3 \\
& & & - & -
\end{tabular}

This general conclusion agrees with other severe cases of low $\mathrm{T}$., not detailed in $(a)$ or $(b)$.

(ii) Some cases detailed in (a) apparently have become arrested. In (b) Case $\mathrm{H}$ had been watched for 19 years and kept good sight till her death in 1930 . 
(iii) On the other hand, a case seen in 1922, not included in $(a)$ because of doubtful diagnosis, is now obviously such an one, and has made steady progress, so that now the $3 / 1000$ field is $12^{\circ}$ around the fixation point, while the other eye now shows an enlarged cup. But one case failed very quickly in a year, the field in the better eye being down to $5^{\circ}$.

(iv) The more carefully a routine examination is made in adults of all cups which are larger and deeper than usual, the larger the number of cases in which the early sign of diminution of the $1 / 1000$ field is found. (Of course, cases that are likely to have this diminished by other causes, such as retinal atheroma $(c)$ or lens haze must be excluded). This, coupled with the fact that severe cases are uncommon, again suggests that many of these slighter cases do not progress far.

It is obvious that the conclusions as to the course of these cases are tentative, except that really severe cases are uncommon; for that strong presumption exists.

Name.-Confusion is arising in nomenclature because of the similarity in the end results in glaucoma and these cases of recession of the nerve head with low tension. I would suggest that " glaucoma " be reserved as formerly for cases of increased tension and the sequelae of the increased tension, independently of whether the tension is due to errors in the amount or composition of the fluid passing into the vitreous, to vascular engorgement, or to hindrances to the removal of the fluid from the eye. For not only are the causes of the phenomena different in increased tension, but the increased tension demands a therapy directed to it, a further reason for not altering the meaning of the word " glaucoma."

Next arises the question of by what name the recession of the nerve head in low tension should be known. By some it is called " glaucoma without pressure" ; but with a different causation this surely is incorrect. In a paper (b) I used the name " cavernous atrophy," borrowing the term from Schnabel's account of certain changes in the nerve head; but this has not caught on, probably because it assumed a pathology for which no proof was produced in the paper referred to.

It should be pointed out the condition called " cavernous atrophy" by Schnabel would fit these cases of recessed cups excellently, whereas it is difficult to see how it can arise in cases of increased tension. The rarefaction of the nerve head tissues followed by contraction, as described by him, would be just the condition which would account for all the symptoms in these low tension cases; both early, before there is any cup enlargement, and later, when contraction has caused the cup to increase. On the other hand, it is difficult to see how an increase of pressure in the eye can cause a rarefaction in the nerve head, or can do anything 
but damage to the tissues by pressure and, nearly always, cause enlargement of the cup. The rarefaction could only exist for the pathologist to find if the pressure were not raised. But from the similarity in the cups and visual effects to glaucoma, may not Schnabel have confused the low tension group with the glaucoma group, and really have described the former?

Although " pseudoglaucoma" has been used for certain congenital conditions of the disc cup, yet it would appear to be for the time being a good name to appropriate to this group of enlarged cups in adults without a rise in the intra-ocular tension; pending an investigation in its pathology, which would probably provide a better. This name suggests its similarities to glaucoma, but distinguishes it from that group of symptoms.

In conclusion I wish to thank Prof. Newman for his help and advice in the compilation of this paper.

\section{REFERENCES}

(a) Pickard, R.-Red field and optic disc resistance in glaucoma and allied conditions. Proc. R. Soc. of Med., Vol. XXIII, Sect. of Ophthal., pp. 57-76, 1930.

(b) Cavernous optic atrophy and its relation to glaucoma. Brit. Jl. of Ophthal., Vol. IX, p. 385, 1925.

(c) The visual field in atheroma of the retinal vessels. Proc. $R$. Soc. of Med., Vol. XIX, Sect. of Ophthal., pp. 55-75, 1926.

(d) Cridland, B.-The tonometer of Schiötz. Brit. Jl. of Ophthal., Vol. I, p. 354, 1917.

\section{A NEW PORTABLE CORNEAL MICROSCOPE}

BY

\section{J. LONGWORTh Blair}

LON DON

IN the diagnosis of certain ophthalmological conditions the necessity for instruments giving high magnification and appropriate lighting has been demonstrated by the popularity of the binocular corneal microscope used in conjunction with some form of slit-lamp. This involves large apparatus which must be fixed. The instrument here described is designed to give a reasonable amount of magnification combined with sufficient illumination in a portable form. The instrument for the purpose of description may be divided into :-

$a$. The microscope and stand. $b$. The source of illumination. The microscope consists of a small optical unit capable of magnification of $\times 10$ to $\times 20$ inclusive, mounted on a stand for application 